\title{
Folded Cavity Surface Emitting Lasers Fabricated by Focused Ion Beam Etching
}

\author{
Guy DeRose, Axel Scherer
}

California Institute of Technology, Electrical Engineering and Applied Physics

1200 E. California Blvd, MC 200-36, Pasadena CA 91125

The development of compact and efficient laser sources at $1500 \mathrm{~nm}$ and $1300 \mathrm{~nm}$ without the need for cleaved edges has been pursued since the early 1980s with the development of vertical cavity surface emitting lasers (VCSELs). These lasers have been widely used for data communications, and are currently applied for high frequency optical interconnects. In the past, much work has been done to define higher power and higher frequency VCSELs. Lithographically defined photonic crystal lasers and annular Bragg reflector lasers have been recently developed within InGaAsP gain material emitting in the telecommunications spectrum. These geometries permitted the reduction of the mode volume to a minimum, but typically suffer from low gain and correspondingly small output power capability. Here, we explore the use of folded cavity lasers to obtain devices that combine large output power with efficient surface emission without the requirement of cleaving short cavities.

Recently, focused ion beam systems have been developed with exquisite resolution and high beam currents. These systems, when used in conjunction with electron beams for alignment of the structures, are ideally suited for defining the angled laser facets that are needed to fold horizontal cavities and convert edge-emitting laser geometries into folded cavity surface emitting lasers (FCSELs) [1,2]. We have developed and optimized recipes for the focused ion beam etching into and through InGaAsP/InP waveguides (Figure 1), and will describe the etch rates (Figure 2), and surface quality of the resulting etched facets. We will compare the performance of edge-emitting lasers with FCSELs, and show the suitability of dual beam focused ion beam and focused electron beam instruments for the purpose of defining high-power surface emitting lasers.

References:

1. H. P. Lee et al., "1.57 $\mu \mathrm{m}$ InGaAs/InP surface emitting lasers by angled focus ion-beam etching," Electronics Letters 28 (1992) 580.

2. J. Purez et al., "Focused ion beam micromachined AlGaAs semiconductor laser mirrors," Electronics Letters 22 (1986) 700.

3. The authors wish to acknowledge funding from the DARPA EPIC program under contract HR0011-04-1-0054. 
Figure 1. SEM micrograph of focused ion beam cut laser facet fabricated with a beam current of 20 $\mathrm{nA}$. This cut required 1 minute of etching time.

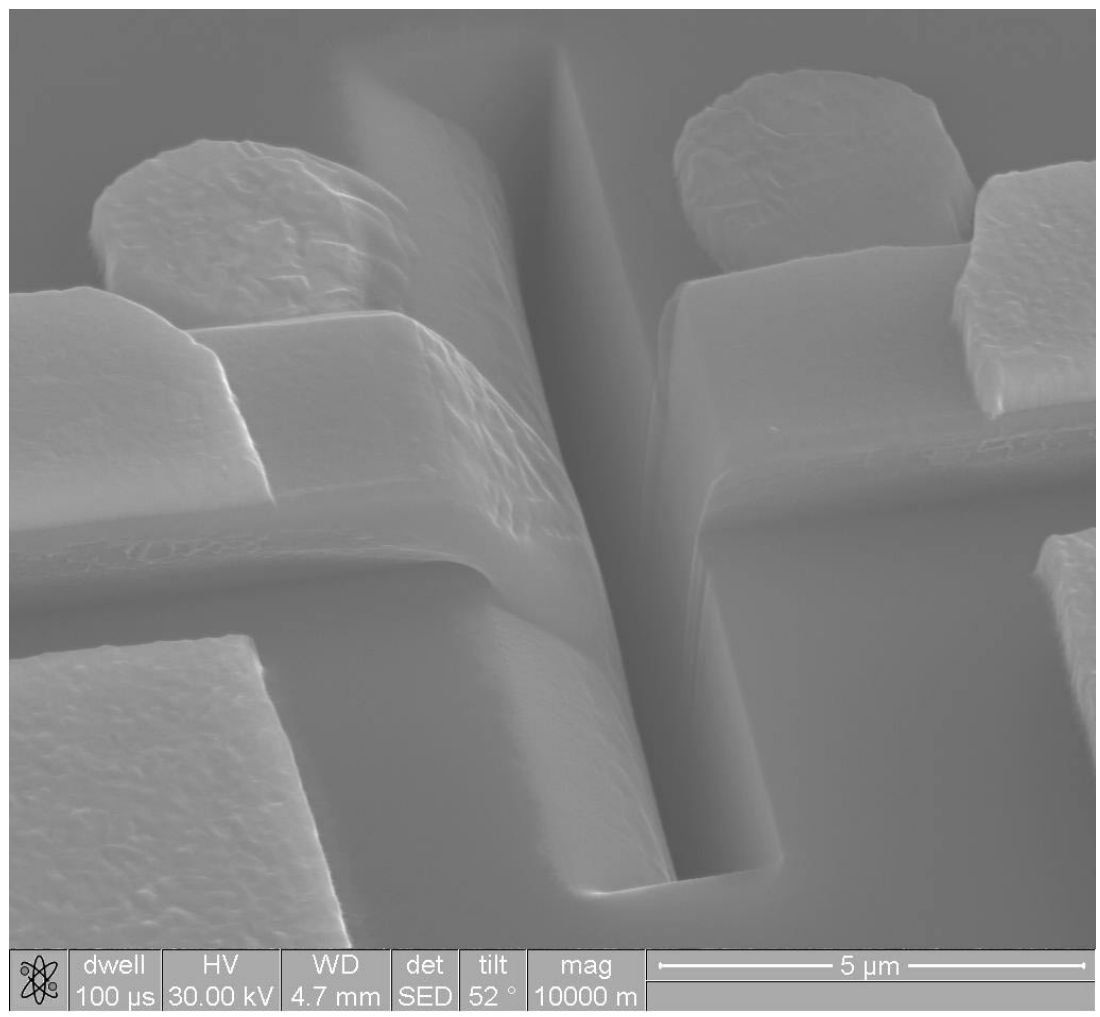

Figure 2. Folded cavity laser mirror etched through InGaAsP waveguide with 4 um depth.

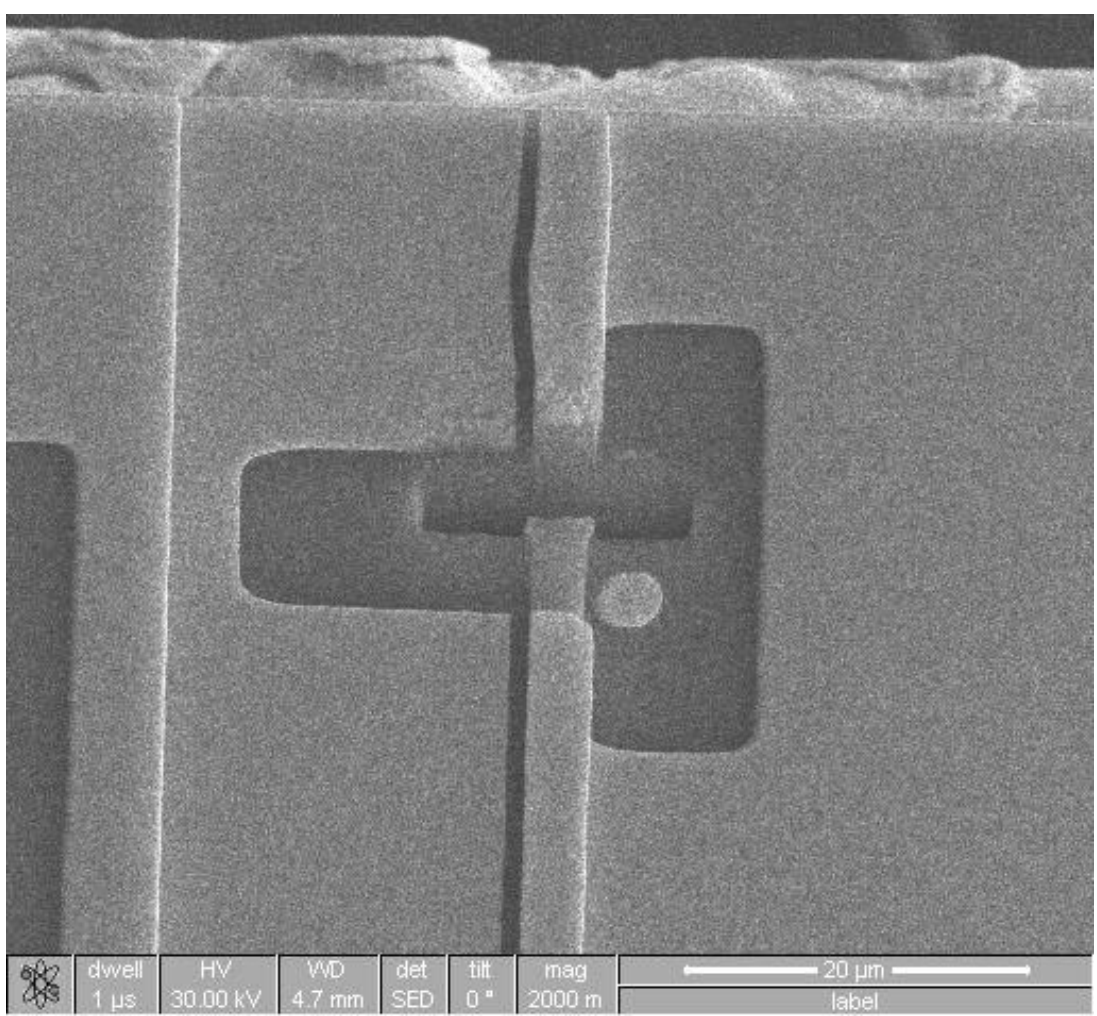

